\title{
Models for Source Structure Corrections
}

\author{
P. Charlot \\ Observatoire de Bordeaux - CNRS/UMR 5804, 2 rue de l'Observatoire, \\ BP 89, 33270 Floirac, France
}

\begin{abstract}
At the milliarcsecond scale, most of the extragalactic radio sources exhibit spatially-extended intrinsic structures which are variable in both time and frequency. Such radio structures set limits on the accuracy of source positions determined with the Very Long Baseline Interferometry (VLBI) technique unless their effects in the astrometric data can be accounted for. We review the modeling scheme for calculating source structure corrections and discuss the magnitude and impact of these effects for the sources that are part of the International Celestial Reference Frame (ICRF). Results obtained by applying source structure corrections to actual VLBI observations on the time-varying source $4 \mathrm{C} 39.25$ $(0923+392)$ are also presented.
\end{abstract}

\section{Theoretical modeling}

The complex visibility $V$ of a spatially-extended source measured by an interferometer with baseline $b$ is given by

$$
V(b, \omega, t)=\int_{\Omega_{s}} I(s, \omega, t) \exp \left(-\frac{i \omega}{c} b \cdot s\right) d \Omega,
$$

where $I(s, \omega, t)$ is the source brightness distribution which depends on the direction $s$ on the sky, the frequency $\omega=2 \pi c / \lambda$, and time $t$, while the integration is over the extended source of solid angle $\Omega_{s}$. If we adopt a reference direction $s_{0}$ within the source, $s$ can be written as $s=s_{0}+\sigma$, where $\sigma$ is in the plane of the sky. The visibility function then can be written as

$$
V(b, \omega, t)=\exp \left(-\frac{i \omega}{c} b \cdot s_{0}\right) \int_{\Omega_{s}} I\left(s_{\mathrm{o}}+\sigma, \omega, t\right) \exp \left(-\frac{i \omega}{c} b \cdot \sigma\right) d \Omega,
$$

which also can be written as

$$
\begin{aligned}
V & =A \exp \left[i\left(\phi_{g}+\phi_{s}\right)\right] \\
& =A \exp \left(i \phi_{t}\right),
\end{aligned}
$$

where the total phase $\phi_{t}$ is the sum of the geometric phase for the reference direction $s_{0}$, 


$$
\phi_{g}=-\frac{\omega}{c} b \cdot s_{0}
$$

and the additional structure phase introduced by the source brightness distribution,

$$
\phi_{s}=\arg \left[\int_{\Omega_{s}} I\left(s_{\mathrm{o}}+\sigma, \omega, t\right) \exp \left(-\frac{i \omega}{c} b \cdot \sigma\right) d \Omega\right] .
$$

The amplitude $A$ observed by the interferometer is given by

$$
A=\left|\int_{\Omega_{s}} I\left(s_{\circ}+\sigma, \omega, t\right) \exp \left(-\frac{i \omega}{c} b \cdot \sigma\right) d \Omega\right| .
$$

The VLBI delay observable used in astrometry is defined by the partial derivative of the total phase with respect to frequency. For an extended source, the delay can be written as

$$
\begin{aligned}
\tau & =\frac{\partial \phi_{t}}{\partial \omega}=\frac{\partial \phi_{g}}{\partial \omega}+\frac{\partial \phi_{s}}{\partial \omega} \\
& =-\frac{1}{c} \boldsymbol{b} \cdot \boldsymbol{s}_{\mathrm{o}}+\tau_{s},
\end{aligned}
$$

where the first term is the geometric delay corresponding to the reference direction $s_{0}$, and the second term $\tau_{s}$ is the additional delay introduced by the extended brightness distribution. Thus, the absolute source position determined in VLBI astrometry is the position of the adopted reference direction $s_{0}$ if delay structure corrections are modeled in this way. In practice, the delay structure corrections $\tau_{s}$ are determined as the slope of a straight line fitted to the individual structure phases calculated for each frequency channel used during the observations, in order to match precisely the scheme used to build the bandwidth synthesis delay observable at the correlator (See Charlot (1990).). The effect introduced by the extended source brightness distribution in the phasedelay rate, defined by the partial derivative of the total phase with respect to time, is obtained in a similar way, and must also be accounted for in a complete astrometric analysis. The interested reader is referred to Charlot (1990) for a more thorough discussion of the phase-delay rate observable.

Calculation of source structure corrections requires the choice of a reference direction $s_{0}$ within the source brightness distribution. As discussed previously, this reference direction is equivalent to the absolute position of the source in the extragalactic reference frame. An appropriate choice of the reference direction is critical for the source position stability since the absolute positions of the source brightness distributions are unknown with respect to the celestial frame. For sources with time-variable structure, it is important that this reference direction be set to a truly kinematically stable morphological feature well identified over time.

In the case of dual-frequency $\mathrm{S} / \mathrm{X}$ observations, the combined $\mathrm{S} / \mathrm{X}$ structure correction is derived by combining the individual X-band and S-band structure corrections with the same scale factors as those used to derive the dualfrequency-calibrated delay from the X-band and S-band delay measurements. 
These factors are approximately 1.08 for the X-band delay and 0.08 for the S-band delay. Such a combination implies indeed that the selected reference directions at X-band and S-band are consistent.

\section{Magnitude of source structure effects}

The theoretical modeling developed in the previous section shows that the source structure corrections depend on the exact form of the spatial brightness distribution of the extended radio source $I(s, \omega, t)$ relative to the geometry of the VLBI baseline vector $b$ projected onto the plane of the sky (see Equations (5) and (7)). The overall magnitude of the source structure effect for a given source is then most easily estimated by calculating these corrections for a range of $u, v$ coordinates (the coordinates $u$ and $v$ are the coordinates of the baseline vector $b$ projected onto the plane of the sky and are expressed in units of the observing wavelength). Following such a scheme, Fey \& Charlot (1997) defined a source "structure index" according to the median value of the structure delay corrections, $\tau_{\text {median }}$, calculated for all projected VLBI baselines that could be possibly observed with Earth-based VLBI (i.e. for all baselines with $\sqrt{u^{2}+v^{2}}$ less than the diameter of the Earth), separating the sources into four classes as follows:

$$
\text { Structure Index }=\left\{\begin{array}{l}
1, \text { if } 0 \mathrm{ps} \leq \tau_{\text {median }}<3 \mathrm{ps}, \\
2, \text { if } 3 \mathrm{ps} \leq \tau_{\text {median }}<10 \mathrm{ps}, \\
3, \text { if } 10 \mathrm{ps} \leq \tau_{\text {median }}<30 \mathrm{ps} \\
4, \text { if } 30 \mathrm{ps} \leq \tau_{\text {median }}<\infty
\end{array}\right.
$$

Based on this definition, two structure indices are obtained for each source, one at X-band and one at S-band, each of which provides an indication of the magnitude of the source structure effect at the corresponding frequency band. For consistency with the procedure used to derive the dual-frequency structure corrections (see above), the structure corrections are scaled by 1.08 at X-band and by 0.08 at S-band, prior to the structure index assignment.

Shown in Figure 1 are contour plots of the radio emission at X-band of four ICRF sources $(0138-097,0108+388,0544+273$ and $2201+315)$ representative of each structure index class. The corresponding structure-effect maps showing the magnitude of the corrections to the VLBI delay observable as a function of the interferometer resolution are also represented along with indication of the mean, rms, median and maximum values of these structure corrections. Figure 1 reflects the increase of the magnitude of the structure effects as the brightness distribution becomes more extended. For $0108+388$, these effects are very large because the source structure is composed of two components of approximately equal strength, causing very low visibility regions in the $u-v$ plane and consequently large structure corrections. (See Charlot (1990) for a detailed study of the case of a two-component model.)

Fey \& Charlot (1997) recommend that only sources with a structure index of either 1 or 2 be used for the most precise astrometric or geodetic work. Sources with a structure index of 3 should only be used with caution while those with a structure index of 4 should not be used at all. 

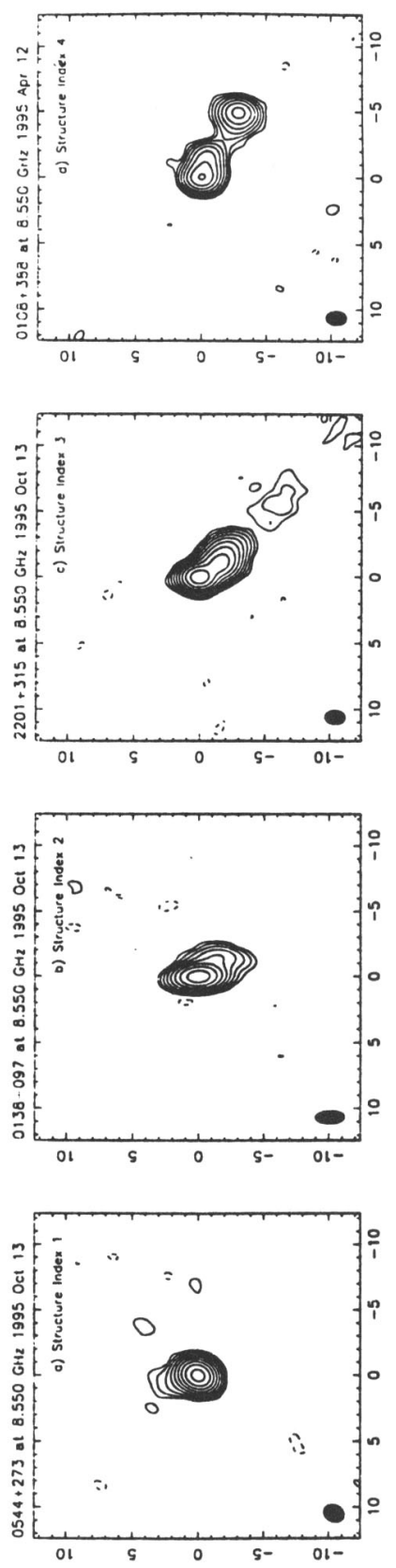
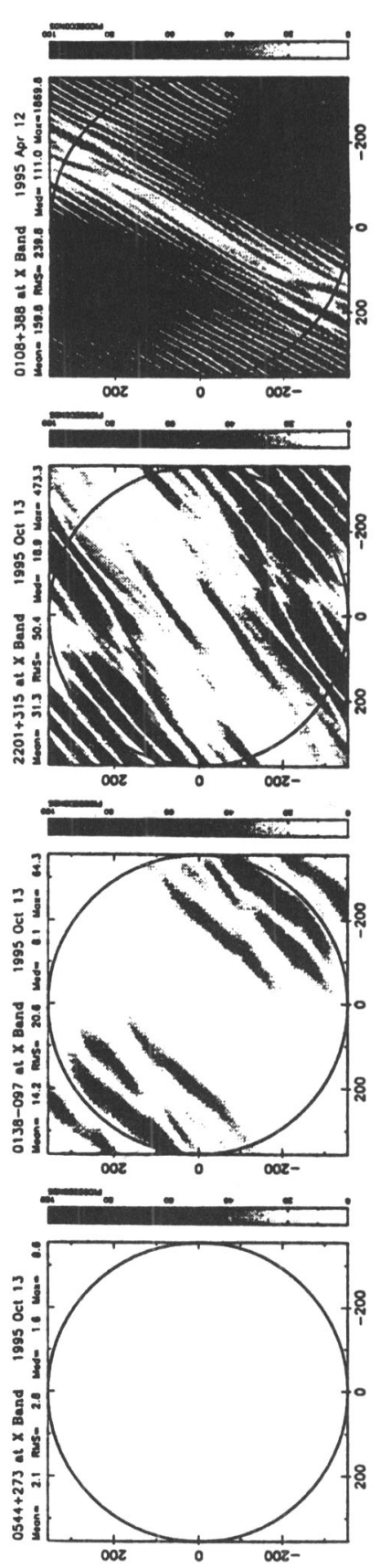

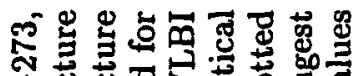

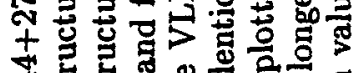

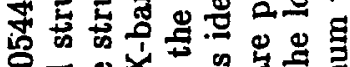

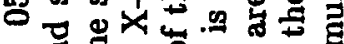

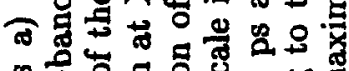

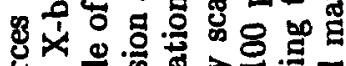

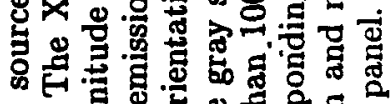

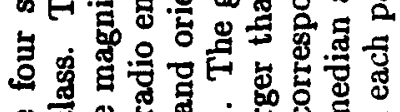

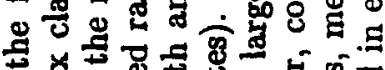

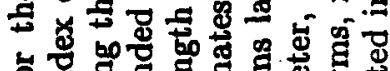

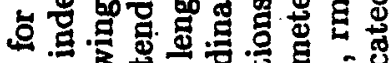

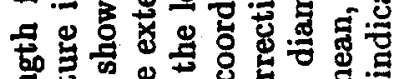
잉

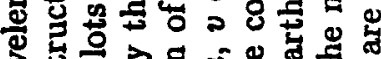

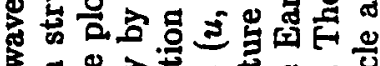

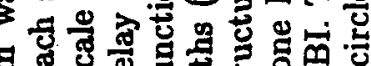

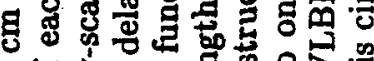

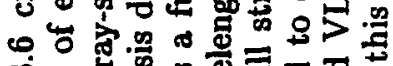

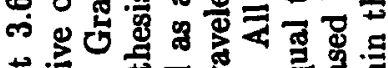

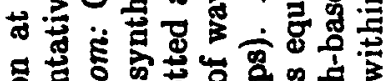

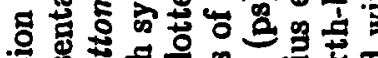

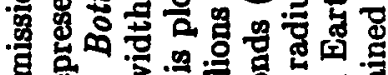
要

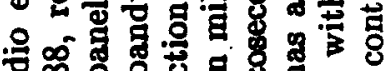

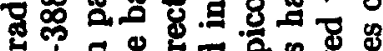

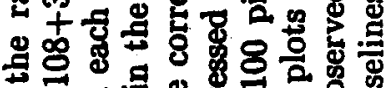
형.

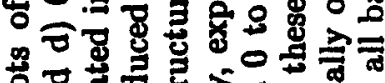

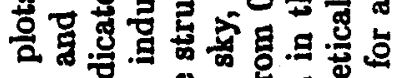

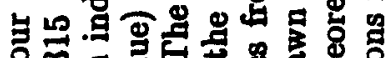

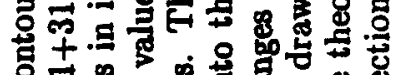

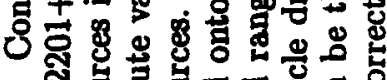
N 今ें0

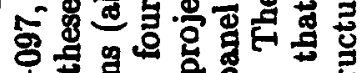
-

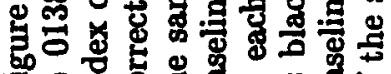

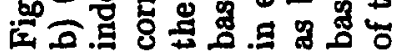



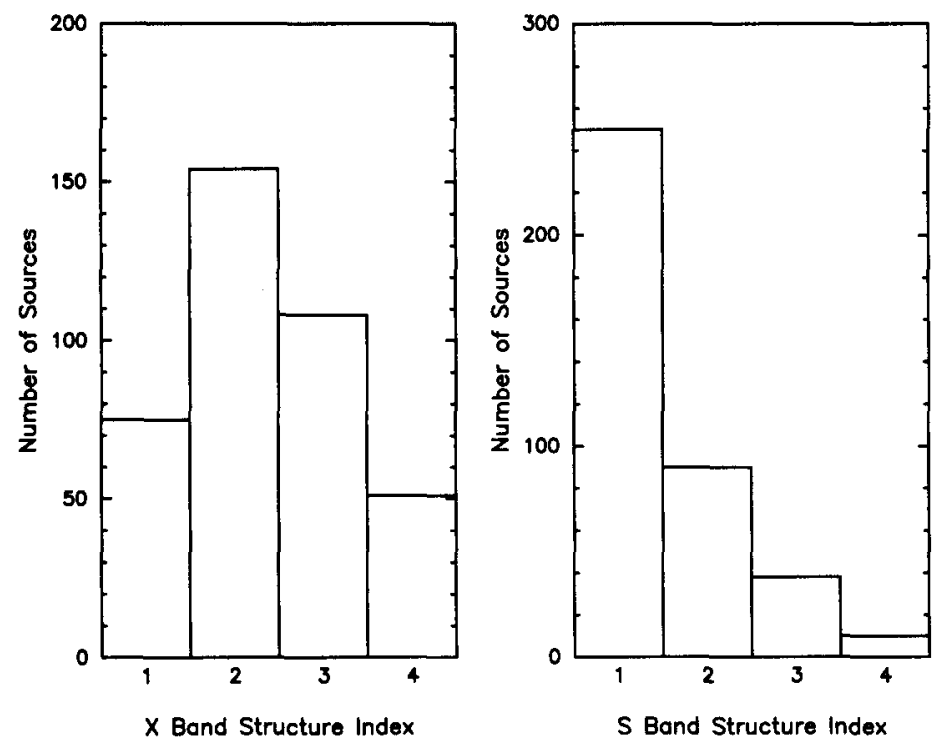

Figure 2. Distribution of the structure index at X-band and S-band for 388 ICRF sources.

\section{The structure index as an indicator for astrometry}

The initial structure index calculation of Fey \& Charlot (1997), which was carried out for 169 sources, has recently been extended for additional sources, bringing the total number of ICRF sources with currently available structure indices to 388 (Fey \& Charlot, 2000), about two-thirds of the ICRF catalog or approximately $90 \%$ of the ICRF catalog north of $-20^{\circ}$ declination. Fey \& Charlot (2000) used this sample to study (i) the structure index distribution in the ICRF, (ii) the relationship between structure index and ICRF source position accuracy, and (iii) the relationship between structure index and ICRF source position stability. Here, we briefly review their results.

\subsection{Structure index distribution in the ICRF}

Figure 2 shows the overall structure index distribution at X-band and S-band for the 388 ICRF sources with currently available structure indices, as reproduced from Fey \& Charlot (2000). At X-band, it is shown that approximately $60 \%$ of the sources in this sample have a structure index of either 1 or 2 , an indication of compact or very compact structures, while the remaining $40 \%$ of the sources with a structure index of either 3 or 4 have more extended emission structures. At S-band, source structure effects appear to be less significant, as reflected by the large number of sources with an S-band structure index of either 1 or 2 in Figure 2 (about $90 \%$ of the sources). This is an indirect indication that the contribution of the S-band structure to the dual-frequency-calibrated delay is usually smaller as compared to the X-band structure contribution, a consequence of the fact that the S-band structure corrections have been scaled by a factor of 0.08 . 

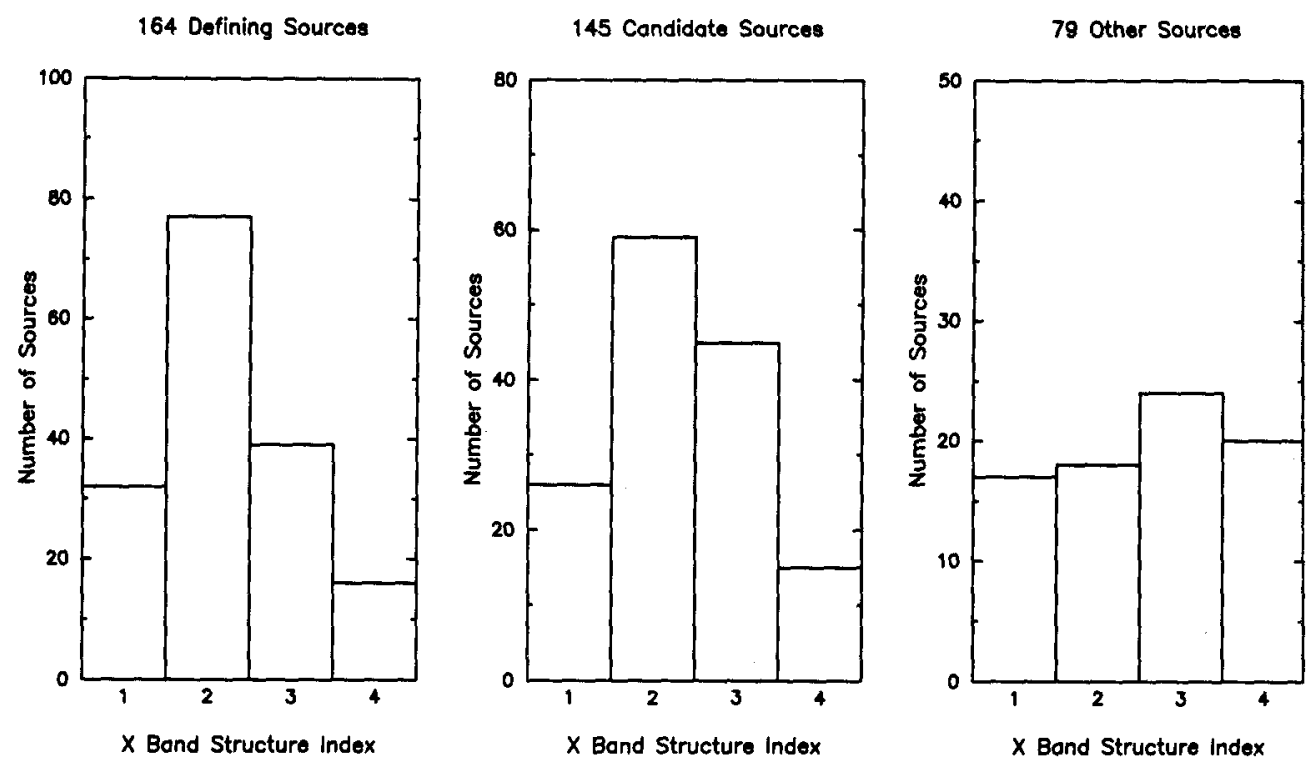

Figure 3. Distribution of the X-band structure index in each ICRF source category. The definition of the three ICRF categories of sources (defining, candidate and "other") is given in Ma et al. (1998) along with the individual source categorization. The 388 ICRF sources with currently available structure indices are included.

In Figure 3, the $\mathrm{X}$-band structure index distribution is compared for each ICRF source category (defining sources, candidate sources, and "other" sources). The difference in distribution between the sources in the defining and "other" sources categories is striking and strongly suggests a causal relationship between the extended intrinsic structure of the "other" sources and their unsuitability for inclusion in the ICRF defining category. The candidate sources appear to have a distribution intermediate between that of the defining sources and the "other" sources categories, which is consistent with the fact that some of the candidate sources could be designated defining sources in a future realization of the ICRF as more data become available or analysis methods improve, whereas others may fall into the "other" sources category. It should also be noted that despite the stringency of the selection criteria for the defining sources (See Ma $e t$ al. (1998).), about one-third of those sources are found to have a structure index of either 3 or 4 , indicating that they are somewhat spatially extended and thus may not be appropriate for defining the celestial frame with the highest level of accuracy. Such sources, especially those with a structure index of 4 (See Fey \& Charlot (2000).), should be given specific attention in the ICRF maintenance process and should be monitored for unexpected behavior as may be anticipated from the value of their structure index.

\subsection{Structure index and ICRF source position accuracy}

Fey \& Charlot (2000) have examined the distribution of uncertainties in right ascension and declination for each X-band structure index class and found that 
the histograms of source position uncertainties progressively deteriorate from an approximately Poisson-like distribution peaked near 0.25 mas for the structure index 1 sources to an almost random distribution for the structure index 4 sources. This deterioration of the position accuracy as the structure index becomes larger is also confirmed when calculating the median and mean uncertainties in right ascension and declination for each X-band structure index class. Table 1 shows that such values increase regularly when the structure index goes from a value of 1 to a value of 4 , which indicates that sources with a larger structure index have larger position uncertainties.

Table 1. $\mathrm{X}$ band Structure Index versus ICRF Position Uncertainty

\begin{tabular}{cccccc}
\hline \hline & & \multicolumn{4}{c}{ Position Uncertainty } \\
$\begin{array}{c}\text { Structure } \\
\text { Index }\end{array}$ & $\begin{array}{c}\text { Number of } \\
\text { Sources }\end{array}$ & \multicolumn{2}{c}{$\begin{array}{c}\text { Mean (mas) } \\
\alpha \cos \delta\end{array}$} & $\delta$ & \multicolumn{2}{c}{$\begin{array}{c}\text { Median (mas) } \\
\end{array}$} & & & & \\
& & & & \\
& 50 & 0.34 & 0.42 & 0.28 & 0.31 \\
1 & 106 & 0.38 & 0.45 & 0.29 & 0.32 \\
2 & 72 & 0.44 & 0.54 & 0.33 & 0.36 \\
3 & 36 & 0.54 & 0.72 & 0.76 & 1.07 \\
4 & & & & & \\
\hline
\end{tabular}

\subsection{Structure index and ICRF source position stability}

Fey \& Charlot (2000) have also searched for any correlation between the time variation of the astrometric coordinates of the sources and the structure index. For this study, they characterized the stability of each source by the weighted rms (wrms) of its position time series (or "arc" source position estimates), as reported by Eubanks (1997), and compared this quantity with the structure index. Table 2 shows that the mean and median values of the "arc" source position wrms are similar for the structure index 1 and 2 classes, but are larger by about 0.05 mas for the structure index 3 class and by $0.15-20$ mas for the structure index 4 class. Thus, in average, the more extended sources are found to have larger position instability than the more compact sources. This is evidence that the source structure size directly correlates not only with the ICRF position accuracy but also with the magnitude of the time variations of the source coordinates.

\section{Modeling source structure effects in actual VLBI observations}

While the structure index is useful for planning experiments and estimating the extra noise caused by source structure in existing data, the ultimate goal is indeed to apply structure corrections directly to actual observations. Progress toward this goal has been made in the case of the extended core-jet source $3 \mathrm{C} 273(1223+026)$, intensively observed during the 80s. Charlot (1994) has shown that modeling the structure effects for this source over approximately a 
Table 2. X band Structure Index versus "Arc" Position wrms

\begin{tabular}{cccccc}
\hline & & \multicolumn{4}{c}{ "Arc" Position wrms } \\
$\begin{array}{c}\text { Structure } \\
\text { Index }\end{array}$ & $\begin{array}{c}\text { Number of } \\
\text { Sources }\end{array}$ & \multicolumn{2}{c}{$\begin{array}{c}\text { Mean (mas) } \\
\alpha \cos \delta\end{array}$} & $\delta$ & \multicolumn{2}{c}{ Median (mas) } \\
& & & & & \\
& & & & \\
\hline 1 & 45 & 0.33 & 0.42 & 0.32 & 0.39 \\
2 & 97 & 0.33 & 0.44 & 0.32 & 0.39 \\
3 & 65 & 0.40 & 0.49 & 0.37 & 0.46 \\
4 & 29 & 0.60 & 0.57 & 0.53 & 0.51 \\
\hline
\end{tabular}

two-year period significantly improves its positional stability over that period. More recently Charlot \& Sovers (2000) have carried out a proper motion analysis of the source 4 C39.25 (0923+392) incorporating source structure models over a 12-yr period. The results of this analysis are reported below.

The source 4 C39.25 is an ideal target for a long-term proper motion study because it has been regularly observed for astrometric and geodetic purposes since the early $80 \mathrm{~s}$. Its structure on milliarcsecond scales consists of three major components approximately aligned along the east-west direction and labeled from east to west as a, b and c (e.g. Shaffer et al., 1987). Two of these components ( $a$ and $c$ ) are found to be stationary with respect to each other, with a separation of about 2 mas, while the third one (b), which emerged from component $\mathrm{c}$ around 1980, moves superluminally toward component a (Shaffer et al., 1987). Later observations have shown that component $b$ has slowed down and become the brighest component as it approaches component a (Alberdi et al., 1993). This evolving morphology is consistent with the VLBI maps shown in Figure 4. These maps have been produced by using VLBI data from the geodetic data base for the purpose of the present study.

The morphology of 4C39.25 is unlike that of a large number of radio sources where superluminal jet components emerge from one side of a strong core and fade afterwards as they move away from the core. For this reason, it is impossible to identify which component $(\mathrm{a} / \mathrm{c}$ or $\mathrm{b})$ is a priori kinematically stable with respect to the absolute celestial frame based only on the observed morphology of 4C39.25. Phase-referenced differential VLBI astrometric observations have solved that question and demonstrated that component $b$ is the one in motion while components $a$ and $c$ are stationary with respect to the absolute celestial frame (Guirado et al., 1995). These results have been further confirmed by Fey et al. (1997) who showed that the observed absolute source motion is consistent with the superluminal motion of the brightest component $b$.

A proper motion analysis similar to that of Fey et al. (1997) with monthly absolute source positions estimated over the period September 1985-March 1997 is shown in Figure 4 (Charlot \& Sovers, 2000). All the observations available from the geodetic and astrometric VLBI data base over that period have been included in this analysis, which was carried out with the JPL VLBI software MODEST (Sovers \& Jacobs, 1996). The results in Figure 4 indicate that the 
right ascension of 4C39.25 increased by about 1 mas between 1985 and 1997 with the bulk of the motion prior to 1992 . This is again consistent with the previously reported deceleration observed for component $b$. An identical analysis incorporating source structure corrections with component a as the reference position is also shown in Figure 4. For this analysis, a simplified representation of the brightness distribution consisting of Gaussian component models has been used to derive source structure corrections at the epochs of the maps in Figure 4, while these models have been interpolated for data at intermediate epochs. This interpolation scheme has allowed us to derive source structure corrections for the complete 12-yr data span. The results of Figure 4 show that the observed proper motion of $4 \mathrm{C} 39.25$ is significantly reduced when incorporating source structure corrections, which confirms directly that the systematic trend in proper motion for 4C3925 is indeed caused by source structure effects. Evidence of the improvement in the position stability is also obtained by comparing the scatter of the monthly position estimates which is reduced from $0.022 \mathrm{~ms}$ to $0.013 \mathrm{~ms}$ when source structure effects are accounted for, while the $\chi^{2}$ value decreases from 12.7 to 6.7 .

Despite this significant improvement, it is noticeable from Figure 4 that the source position instability is not entirely eliminated. This may be due to insufficient time sampling of the images in Figure 4 or to uncertainties in the identification of the reference position at each epoch. Additional maps at intermediate epochs are being made to improve these results further.

\section{Conclusion}

The analyses carried out so far to evaluate the magnitude of the source structure effects and their impact on the ICRF indicate that these effects are significant. For the bandwidth synthesis delay, they range from a few picoseconds for the most compact sources to several nanoseconds for the very extended sources. The structure index defined by Fey \& Charlot $(1997,2000)$ is a useful indicator to evaluate the source quality. Additionally, it has been demonstrated that the observed absolute proper motion of the source $4 \mathrm{C} 39.25$ can be largely reduced when correcting for structure effects (Charlot \& Sovers, 2000). Such studies should be pursued further in the future, in particular by using the Radio Reference Frame Image Data Base from the U.S. Naval Observatory. Future prospects with this data base include source structure index monitoring and astrometric analyses incorporating structure corrections for a complete sample of sources. 

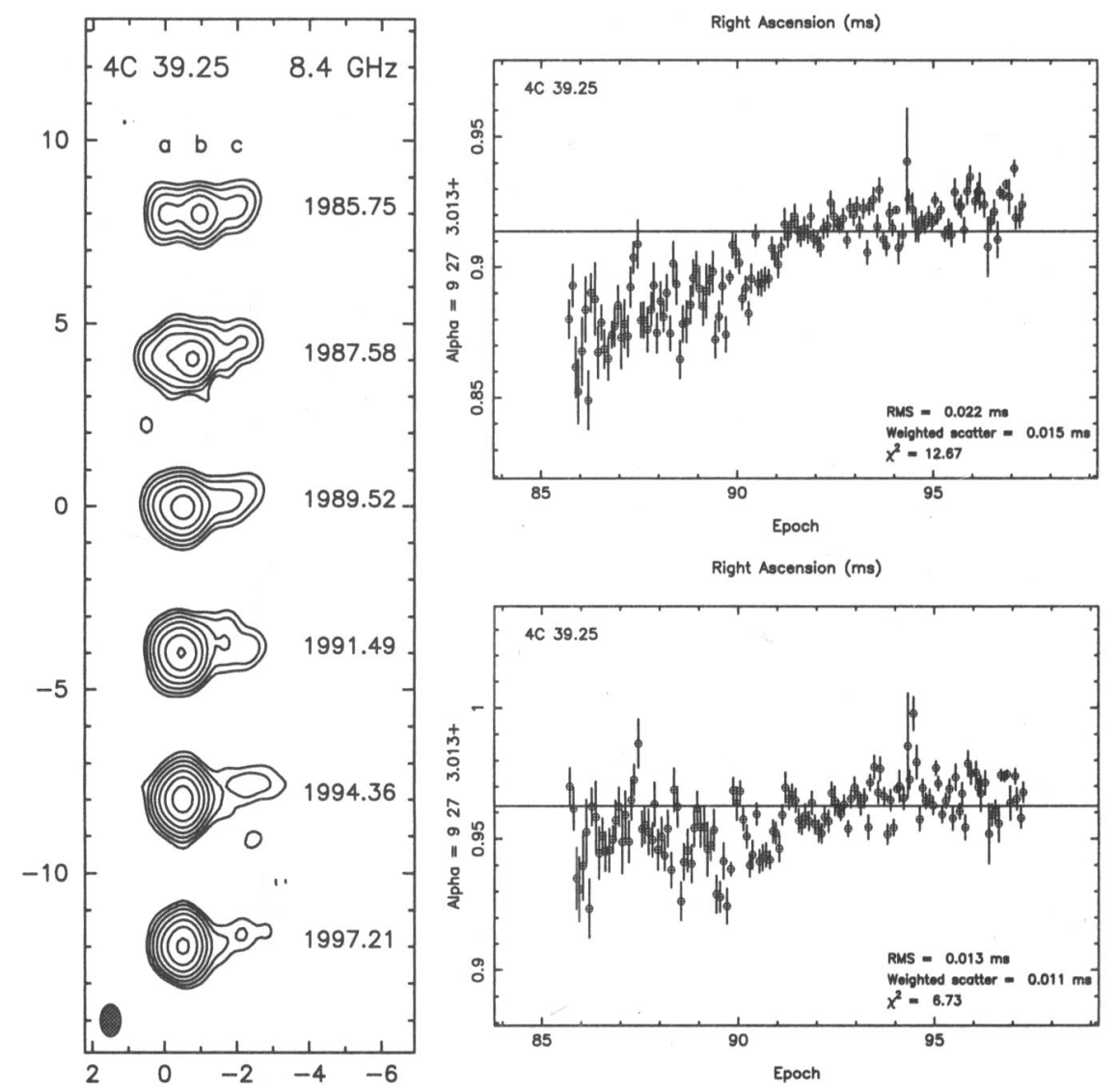

Figure 4. Left: Contour plots of the radio emission at $3.6 \mathrm{~cm}$ wavelength of the source $4 \mathrm{C} 39.25$ at 6 epochs. The three major components are labeled $\mathrm{a}, \mathrm{b}$ and $\mathrm{c}$ in the first image. Top right: Estimated monthly positions in $\alpha$ of 4 C39.25 over the period September 1985-March 1987 without modeling source structure effects. Bottom right: Estimated monthly positions in $\alpha$ of $4 \mathrm{C} 39.25$ over the same period when source structure are modeled with component a as the reference position. 


\section{References}

Alberdi, A., Marcaide, J. M., Marscher, A. P., Zhang, Y. F., Elósegui, P., Gómez, J. L., Shaffer, D. B., 1993, Astrophys. J., 402, 160.

Charlot, P., 1990, Astron. J., 99, 1309.

Charlot, P., 1994, in VLBI Technology: Progress and Future Observational Possibilities, eds. T. Sasao, S. Manabe, O. Kameya, \& M. Inoue, Tokyo: Terra Scientific Publishing Company, 287.

Charlot, P. \& Sovers, O. J., 2000 (in preparation).

Eubanks, T. M., 1997, 23rd Meeting of the IAU, Joint Discussion 7.

Fey, A. L. \& Charlot, P., 1997, Astrophys. J. Sup., 111, 95.

Fey, A. L. \& Charlot, P., 2000, Astrophys. J. Sup., (in press).

Fey, A. L., Eubanks, T. M., \& Kingham, K. A., 1997, Astron. J., 114, 2284.

Guirado, J. C., Marcaide, J. M., Alberdi, A., Elósegui,, P., Ratner, M. I., Shapiro, I. I., Kilger, R., Mantovani, F., Venturi, T., Rius, A., Ros, E., Trigilio, C., Whitney, A. R., 1995, Astron. J. 110, 2586.

Ma, C., Arias, E. F., Eubanks, T. M., Fey, A. L., Gontier, A.-M., Jacobs, C. S., Sovers, O. J., Archinal, B. A., Charlot, P., 1998, Astron. J., 116, 516.

Shaffer, D. B., Marscher, A. P., Marcaide, J., Romney, J. D., 1987, Astrophys. $J ., 314, \mathrm{~L} 1$.

Sovers, O. J., \& Jacobs, C. S., 1996, Observation Model and Parameter Partials for the JPL VLBI parameter Estimation Software "MODEST", Jet Propulsion laboratory Publ. 83-89, Rev. 6, Pasadena, CA. 